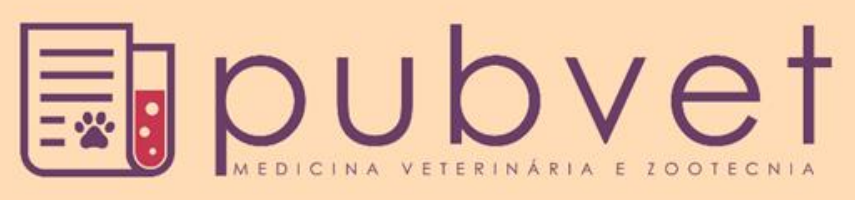

https://doi.org/10.31533/pubvet.v15n04a781.1-6

\title{
Contribuição do exame ultrassonográfico como auxílio diagnóstico de leiomioma intestinal em cão: Relato de caso
}

\author{
Luís Augusto Macedo Gonçales $^{1 \bullet}$, Valéria Silva de Podestá $^{1 \bullet}$, Daniela Loureiro Henrique ${ }^{2 * \bullet}$, \\ Pietra Bento de Capitani ${ }^{3}$
}

${ }^{1}$ Médico veterinário autônomo

${ }^{2}$ Mestre em Medicina Veterinária e Bem-Estar Animal - Universidade Santo Amaro - UNISA-São Paulo, Brasil

${ }^{3}$ Discente em Medicina Veterinária- Universidade Metodista de São Paulo - UMESP-São Bernardo do Campo, Brasil

*Autor para correspondência, E-mail: daniela@pataepatela.com.br

\begin{abstract}
Resumo. Doenças com quadros obstrutivos, inflamatórios, parasitários e neoplásicos estão entre as patologias gastrointestinais que acometem cães e gatos, contudo, somente as manifestações clínicas, muitas vezes são insuficientes e subjetivas, e podem não contribuir para diferenciar lesões intestinais inflamatórias de neoplasias intestinais, sendo o exame ultrassonográfico a modalidade de diagnóstico mais eficiente e menos invasiva para detecção de tumores gastrointestinais em cães e gatos. Além disso, a ultrassonografia pode auxiliar a biópsia do segmento acometido, que pode ser realizada também por endoscopia ou ocasionalmente por laparotomia exploratória para obtenção do diagnóstico definitivo. O objetivo deste relato é salientar a importante contribuição do exame ultrassonográfico para o auxílio diagnóstico de neoplasias intestinais em cães e gatos, descrevendo um caso de neoformação duodenal (leiomioma) em um paciente da espécie canina.
\end{abstract}

Palavras-chave: Intestino, neoplasia, obstrução, ultrassom, vômitos

\section{Contribution of ultrasound examination to the diagnostic aid of intestinal leiomyoma in a dog: Case report}

\begin{abstract}
Obstructive diseases, inflammatory and neoplastic parasitic frames are among the diseases that affect the gastrointestinal small animals, but only clinical signs are often insufficient and subjective and can't differentiate contribute to inflammatory bowel lesions of intestinal tumors, and the ultrasonographic mode more efficient and less invasive diagnosis for detection of gastrointestinal tumors in dogs and cats. In addition, ultrasound can facilitate biopsy of the affected segment can also be performed by gastroduodenoscopy or occasionally by laparotomy for the definitive diagnosis. The objective of this report is to describe a case of duodenal leiomyoma in a patient's canine species, in addition to stress the important contribution of ultrasonography to aid diagnosis of neoplasms of the gastrointestinal tract in small animals.
\end{abstract}

Keywords: Intestine, neoplasia, obstruction, ultrasound, vomiting

\section{Contribución de la ecografía como ayuda diagnóstica del leiomioma intestinal en un perro: Reporte de caso}

Resumen. Las enfermedades con afecciones obstructivas, inflamatorias, parasitarias y neoplásicas se encuentran entre las patologías gastrointestinales que afectan a perros y gatos, sin embargo, solo las manifestaciones clínicas suelen ser insuficientes y subjetivas, pudiendo no contribuir a diferenciar las lesiones inflamatorias intestinales de las neoplasias 
intestinales, siendo la ecografía la modalidad de diagnóstico más eficaz y menos invasiva para detectar tumores gastrointestinales en perros y gatos. Además, la ecografía puede ayudar a la biopsia del segmento afectado, que también se puede realizar mediante endoscopia u ocasionalmente mediante laparotomía exploradora para obtener el diagnóstico definitivo. El propósito de este informe es resaltar la importante contribución de la ecografía a la ayuda diagnóstica de neoplasias intestinales en perros y gatos, describiendo un caso de neoformación duodenal (leiomioma) en un paciente canino.

Palabras clave: Intestino, neoplasia, obstrucción, ecografía, vómitos

\section{Introdução}

As principais afecções que acometem o trato gastrointestinal (TGI) de pequenos animais são doenças de origens obstrutivas, inflamatórias, parasitárias e neoplásicas. As manifestações clínicas inespecíficas, frequentemente encontradas em doenças de origens inflamatórias e neoplásicas, são muitas vezes insuficientes para diferenciação entre elas, especialmente no caso de enfermidades inflamatórias crônicas (Froes et al., 2007; Penninck \& D’Anjou, 2011).

A ultrassonografia abdominal é um método não-invasivo de avaliar a arquitetura, parênquima e tamanho dos órgãos abdominais, e no caso do trato gastrointestinal, esta modalidade diagnóstica permite uma avaliação dinâmica da motilidade, progressão do conteúdo, além de permitir a mensuração da espessura da parede e visualização da estratificação das camadas sem o uso de radiação ionizante, anestesia geral ou preparação especial do paciente (Penninck \& D’Anjou, 2011). Contudo, segundo Gaschen (2013), na maioria dos casos, o exame ultrassonográfico do trato gastrointestinal deve ser interpretado em conjunto com os achados de radiografias abdominais, sendo que a mesma fornece uma visão geral do abdome que o exame ultrassonográfico não permite, podendo auxiliar no entendimento das características ultrassonográficas que estão sendo observadas.

\section{Aspectos neoplásicos gerais do leiomioma}

Os leiomiomas são achados tipicamente incidentais em estômago de pacientes geriátricos, podendo ser encontrados ocasionalmente em segmentos intestinais (Penninck, 2004). Um estudo realizado por Penninck \& D'Anjou (2011) revelaram que nenhuma das neoplasias do músculo liso relatadas apresentou linfadenopatia regional.

Em humanos, o leiomioma é a lesão benigna sintomática mais comum do intestino delgado, sendo usualmente encontrada no jejuno e íleo e podem apresentar quatro formas de crescimento na parede intestinal: extramural, intramural, extra luminal e intraluminal. Sabe-se que, neoplasias gastrointestinais do músculo liso são incomuns na rotina clínica veterinária, mas podem representar até um terço de todas neoplasias intestinais caninas (Myers III \& Penninck, 1994) sendo a intussuscepção uma complicação rotineira nessas patologias (Penninck, 2004).

Segundo Froes et al. (2007) e Penninck \& D'Anjou (2011), sonograficamente os leiomiossarcomas podem apresentar-se como grandes massas parietais extrínsecas, heterogêneas, com áreas hipo/anecogênicas, notando-se perda de estratificação das camadas. Efusão peritoneal pode ser visualizada.

As neoplasias estromais gastrointestinais caninos (GISTs) são um subtipo recente de neoplasia de células fusiformes gastrointestinais (Figura 1), reconhecidas pelo uso crescente de imunohistoquímica (Hobbs et al., 2015). Segundo Bergman (2013), as neoplasias estromais gastrointestinais (GIST) são mais comumente observadas em intestino grosso, contudo, são descritas com menor frequência em intestino delgado e no estômago.

Em um estudo realizado por Frost et al. (2003), cinquenta cães com neoplasias mesenquimais foram avaliados para determinar a ocorrência de leiomiomas e neoplasias estromais gastrointestinais, comparando suas características clinicopatológicas. Vinte e uma neoplasias (42\%) foram histologicamente reclassificados como neoplasias estromais gastrointestinais (GISTs) e 29 neoplasias (58\%) como Leiomiomas, com base na sua semelhança histológica com neoplasias humanas homólogas. Em referido estudo, os GISTs ocorreram igualmente em machos e fêmeas, com idade média de 11 anos 
(variação de 5 a 14 anos). Cinco GISTs (24\%) foram associados a manifestações clínicas e seis (29\%) apresentaram metástases no fígado ou na cavidade abdominal. Os GISTs ocorreram no intestino grosso $(10)=48 \%$, intestino delgado $(6)=29 \%$, estômago $(04)=19 \%$ e mesentério do intestino delgado $(1)=$ $5 \%$.

Em humanos, características ultrassonográficas dos GISTs incluem uma grande neoplasia intratramural que surge da camada muscular, que raramente afeta a camada mucosa, regiões de cavitação, e adicionalmente, as características que podem sugerir alto potencial maligno, que foram o tamanho e heterogenicidade interna com presença de áreas hipoecogênicas intratumorais (Wronski et al., 2009). Na medicina veterinária, as características ultrassonográficas das neoplasias de músculo liso incluem massas excêntricas com cavitações; porém, tais carcterísticas foram descritas por Myers III \& Penninck (1994), antes de uma melhor compreensão dos GISTs.

Segundo Hobbs et al. (2015), as características ultrassonográficas dos GISTs confirmados de fato, ainda não foram claramente descritas, e em referido estudo, os achados indicaram que a diferenciação entre GISTs e não GISTs em cães, não é possível baseado apenas nas características ultrassonográficas, no entanto, o ultrassom auxiliou na localização e na determinação do grau de envolvimento das neoplasias de células fusiformes gastrointestinais (Figura 1).

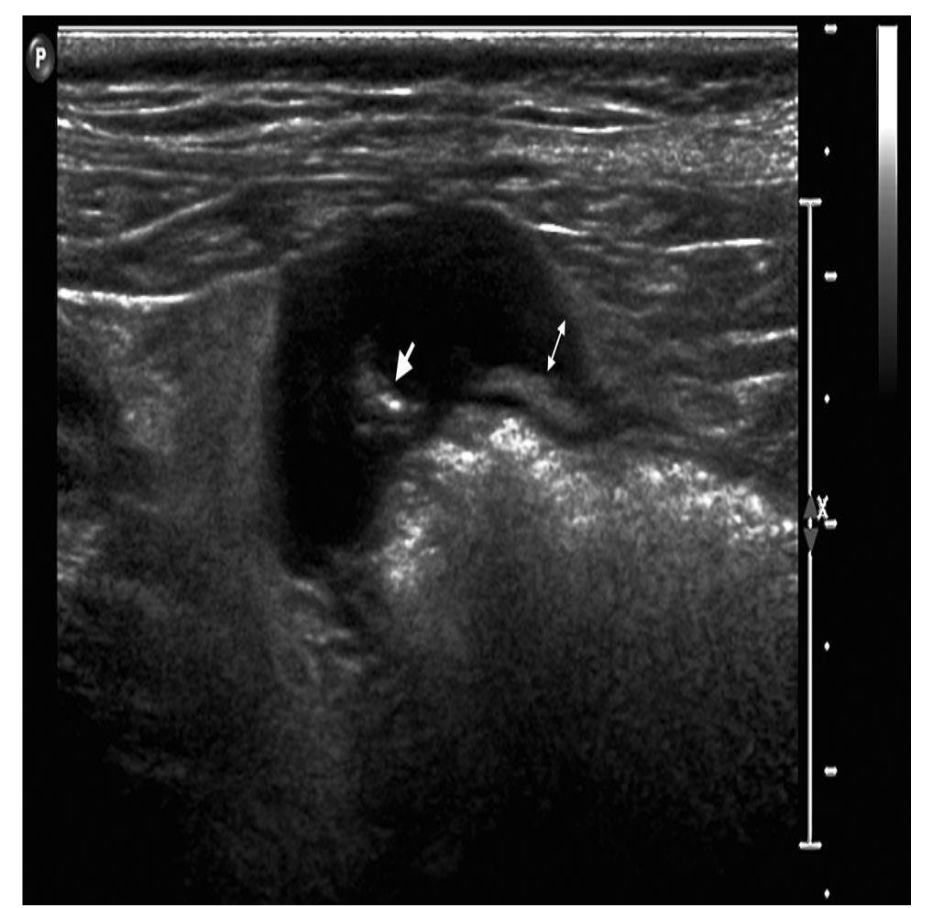

Figura 1. Ultrassonografia sagital de uma neoplasia cecal, posteriormente confirmado como GIST, em porção mais distal do ceco . Lúmen representado pela seta branca e camada muscular representada pela seta branca de duas pontas (Hobbs et al., 2015).

\section{Relato de caso}

Foi atendido um paciente da espécie canina, da raça Poodle, macho, 14 anos, que segundo o proprietário, apresentava episódios eméticos repetitivos (cerca de 3 ou 4 ao dia). Proprietário relatou que percebeu emagrecimento do animal, porém, somente notou a hiporexia nos últimos 3 dias. Após questionado, descreveu que notou discreta diminuição na frequência de defecação e no volume das fezes. Ao exame clínico, o paciente apresentou temperatura retal de $39,0^{\circ} \mathrm{C}$, desidratação $(6 \%$ a $7 \%)$, mucosa oral levemente hipocorada, presença de periodontite, retração gengival e gengivite em caninos, ausência de sialorreia e sensibilidade acentuada à palpação abdominal, o que dificultou a avaliação devido à tensão apresentada pelo paciente. O paciente ainda apresentava taquisfigmia e taquicardia leves, apatia, pouco responsivo, supostamente devido à dor, sendo internado após o atendimento clínico, mas a proprietária negou a realização de exames, tendo autorizado somente o tratamento paliativo. 
No dia seguinte, apesar do controle emético alcançado, não houve mudança substancial no quadro geral do paciente, sendo novamente reafirmada a necessidade de exames, agora autorizados pela proprietária. Foram realizados exames hematológicos, obtendo-se os seguintes resultados: glicemia sérica: $55 \mathrm{mg} / \mathrm{dl}(70-110 \mathrm{mg} / \mathrm{dl}$.), hematócrito: $52 \%$ (37-55\%), leucocitose por neutrofilia: $20.792 / \mu \mathrm{l}$ (6000-17000/ $\mu \mathrm{l})$, contagem plaquetária: $215.000 / \mu \mathrm{l}(200.000-500.000 / \mu \mathrm{l})$, presença de diversos neutrófilos pouco segmentados e tóxicos, creatinina: $1,1 \mathrm{mg} / \mathrm{dl}(0,5-1,5 \mathrm{mg} / \mathrm{dl})$, ureia: $45 \mathrm{mg} / \mathrm{dl}(21$ 59mg/dl), ALT: 122U/L (21-102U/L); FA: 67U/L (20-156U/L), albumina sérica: 2,4g/dL (2,6 -3,3 g/dL).

Considerando-se os aspectos clínicos e laboratoriais, suspeitou-se de gastrite, hepatopatia, pancreatite, obstrução por ingestão de corpo estranho ou neoplasia, optando-se pelo exame ultrassonográfico devido a possibilidade de avaliar os principais segmentos do trato gastrintestinal, assim como observar a topografia, motilidade, conteúdo, espessamento da parede, integridade da estratificação das camadas e adjacências, bem como o parênquima de órgãos como o fígado e o pâncreas, colaborando de forma significativa para com o diagnóstico, terapêutica e prognóstico.

O exame ultrassonográfico abdominal exploratório avaliou todos os órgãos da cavidade abdominal, porém, devido as suspeitas clínicas, foi dada ênfase na avaliação do trato gastrointestinal. Foi utilizada janela intercostal cranial direita para a adequada visibilização do segmento proximal de duodeno descendente o qual apresentou uma formação focal com superfície irregular, contorno parcialmente definido e formato anelar, medindo em sua totalidade cerca de $3,40 \mathrm{~cm}$ x $3,20 \mathrm{~cm}$. Em referida formação, notou-se parede intestinal acentuadamente espessada medindo cerca de $1,60 \mathrm{~cm}$, com perda da estratificação das camadas, ecogenicidade mista predominantemente hipoecogênica com aspecto heterogêneo e ecotextura grosseira (Figura 2).

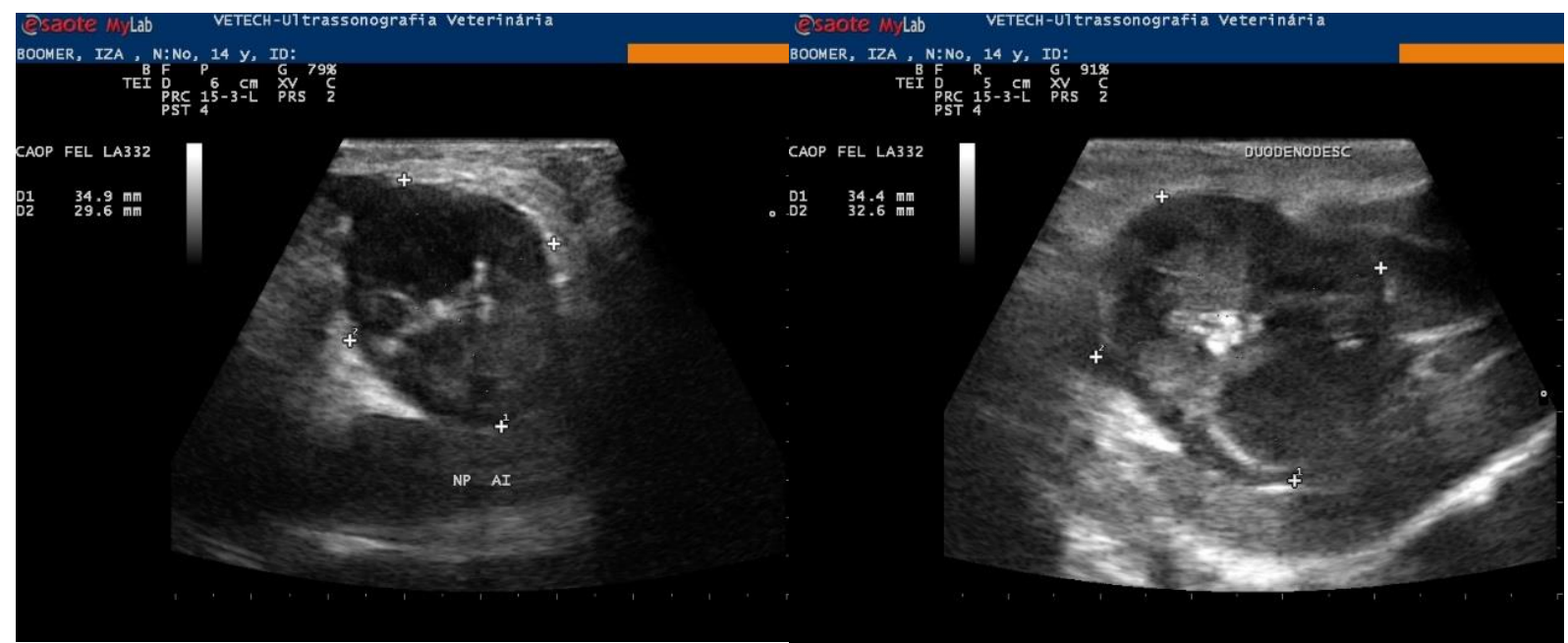

Figura 2. Imagens ultrassonográficas de um corte transversal de um segmento cranial de duodeno descendente proximal, apresentando espessamento focal com perda de estratificação das camadas. Imagens obtidas com transdutor de 7,5 MHz. Arquivo pessoal do autor.

Os linfonodos regionais não estavam evidentes. Ramo pancreático direito não apresentava alterações sonográficas. Notou-se ainda o estômago repleto por conteúdo anecogênico (líquido) e gasoso (reverberação), dificultando a avaliação de corpo e ramo pancreático esquerdo, contudo, evidências de alterações peripancreática, como peritonite focal e/ou efusão, não foram encontradas. Ramo pancreático direito não apresentava alterações sonográficas; porém, o segmento de duodeno descendente caudal a neoformação, apresentava parede espessada, medindo cerca de $5,5 \mathrm{~mm}$ com a estratificação das camadas preservadas, além de um moderado pregueamento, sugestivo de enterite focal. A motilidade dos segmentos intestinais encontrava-se dentro da normalidade (4 movimentos por minuto), porém a motilidade gástrica estava reduzida (entre 1 e 2 movimentos por minuto). Demais segmentos de alças intestinais sem alterações sonográficas dignas de nota.

Após o exame, concluiu-se que as alterações sonográficas encontradas eram sugestivas de processo neoplásico, corroborando com Penninck \& D'Anjou (2011) os quais relataram que os cães com enterite apresentaram lesões com uma espessura média de parede de $6,0 \mathrm{~mm}$, o que foi significativamente menor 
do que a espessura média da parede de cães com tumores intestinais (1,5 centímetros). Esta significativa diferença pode indicar que os tumores intestinais tendem a apresentar espessamento da parede mais acentuados do que no processo inflamatório. Massas neoplásicas no intestino são geralmente focais ou multifocais enquanto a enterite normalmente tem envolvimento mais difuso, apresentando na maioria das vezes, a estratificação das camadas preservada e espessamentos menos acentuados. Em nenhum momento considerou-se a pitiose como um possível diagnóstico diferencial, contudo ficou esclarecido que o diagnóstico definitivo seria obtido através de exame histopatológico.

Devido ao quadro obstrutivo em duodeno, independente do diagnóstico conclusivo, o paciente foi encaminhado para procedimento cirúrgico, o qual revelou uma neoformação focal com aspecto circunferencial localizada em segmento cranial de duodeno descendente, bem próximo ao piloro, correspondendo aos achados ultrassonográficos. Durante o procedimento cirúrgico optou-se pela técnica de enterectomia em cunha, onde permitiu-se a retirada sem margem de segurança, de aproximadamente $70 \%$ do segmento acometido uma vez que a enterectomia do segmento total era contraindicada devido à proximidade ao pâncreas.

Após procedimento cirúrgico, o material foi enviado para exame histopatológico o qual determinou o diagnóstico definitivo de Leiomioma para a formação intestinal (igura 3).

O paciente não apresentou melhoras nos episódios de êmese, vindo a óbito no terceiro dia pósoperatório sendo que o proprietário não autorizou exames ultrassonográficos para monitoramento e nem a necropsia.

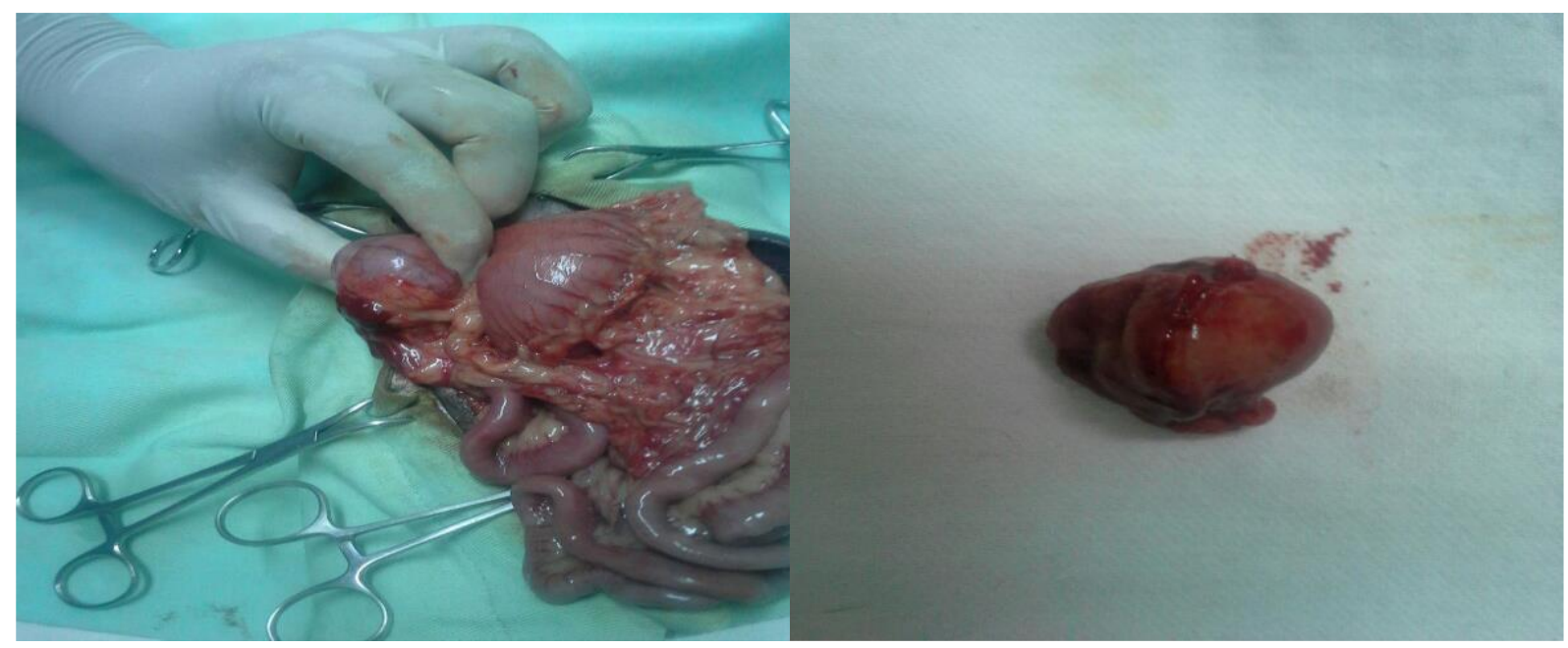

Figuras 3. Neoformação removida e encaminhada para histopatológico. Segmento de duodeno descendente proximal acometido.

\section{Resultados e discussão}

O exame ultrassonográfico é a modalidade de diagnóstico mais eficiente e menos invasiva para detecção de neoplasias gastrintestinais em cães e gatos, sendo rotineiramente requerido em pacientes suspeitos de neoplasias gastrointestinais e muitas vezes é suficiente para eliminar diagnósticos diferenciais, principalmente a doença intestinal inflamatória crônica, contudo, sugere-se sempre que possível, associar ao exame radiográfico abdominal simples.

Existem inúmeras neoplasias que podem acometer o trato digestório, em sua maioria malignas. O estadiamento das lesões é fundamental para avaliar o grau de disseminação das neoplasias. Em geral, não há predisposição sexual para o aparecimento das lesões e, assim como as demais manifestações oncológicas, as neoplasias gastrointestinais são mais frequentes em animais senis (Hugen, 2017). Rotineiramente, utiliza-se a abordagem abdominal ventral para avaliação do TGI, contudo, alguns pontos anatômicos requerem atenção especial no momento do exame devido sua localização, optandose por janelas alternativas, como a janela intercostal direita utilizada no presente relato para melhor avaliação da região proximal de duodeno descendente e piloro.

Apesar de o leiomioma tratar-se de uma neoplasia benigna, dependendo da localização no trato intestinal, pode ocasionar quadros obstrutivos, intussuscepção e complicações hemorrágicas e 
sistêmicas (ulcerações, peritonite e septicemia) devido sua ruptura. Enterites com infiltrados linfoplasmocíticos e linfoma do intestino delgado apresentam características ultrassonográficas similares.

Considera-se que a gravidade do espessamento avaliada isoladamente não é característica confiável para distinguir diferentes doenças inflamatórias das neoplasias, tornando-se de fundamental importância avaliar a estratificação de camadas, bem como a extensão de lesão ou a camada acometida, tornando tais parâmetros valiosos para distinguir neoplasia de inflamação. A avaliação da aparência das camadas da parede do TGI é útil para determinar a natureza e localização de processos patológicos.

Apesar de, na grande maioria dos casos de neoplasias gastrointestinais, o espessamento acentuado da parede associado à perda de estratificação das camadas seja altamente preditivo, deve-se ainda assim, considerar a possibilidade de outras afecções com aspectos sonográficos semelhantes como as enterites crônicas, pitiose gastrintestinal e a enterite granulomatosa.

\section{Conclusão}

O exame ultrassonográfico é um exame de triagem, assim como os exames laboratoriais, que direcionam o diagnóstico e consequentemente auxiliam o médico veterinário a definir sua conduta e estabelecer o melhor tratamento para seus pacientes. Apesar do exame ultrassonográfico ser menos invasivo e imprescindível para avaliação prévia a procedimentos endoscópicos ou cirúrgicos, concluise que, o diagnóstico definitivo das alterações ultrassonográficas, principalmente do trato gastrointestinal, só pode ser realizado por meio de biópsias e avaliação histopatológica.

\section{Referências}

Bergman, P. J. (2013). Neoplasia. Small intestine. In R. J. Washabau \& M. J. Day (Eds.), Canine and feline gastroenterology (pp. 651-728). Elselvier Saunders.

Froes, T. R., Iwasaki, M., Campos, A. G., Torres, L. N., \& Dagli, M. L. Z. (2007). Avaliação ultrasonográfica e pelo Doppler colorido do carcinoma de células transicionais da bexiga em cães. Arquivo Brasileiro de Medicina Veterinária e Zootecnia, 59(6), 1400-1407.

Frost, D., Lasota, J., \& Miettinen, M. (2003). Gastrointestinal stromal tumors and leiomyomas in the dog: a histopathologic, immunohistochemical, and molecular genetic study of 50 cases. Veterinary Pathology, 40(1), 42-54. https://doi.org/10.1354/vp.40-1-42.

Gaschen, L. (2013). Ultrassonographic Imaging of the gastrointestinal tract. In R. J. Washabau \& M. J. Day (Eds.), Diagnostic Imaging of gastrointestinal tract (pp. 228-235). Elsevier.

Hobbs, J., Sutherland-Smith, J., Penninck, D., Jennings, S., Barber, L., \& Barton, B. (2015). Ultrasonographic features of canine gastrointestinal stromal tumors compared to other gastrointestinal spindle cell tumors. Veterinary Radiology \& Ultrasound, 56(4), 432-438. https://doi.org/10.1111/vru.12253.

Hugen, G. F. G. P. (2017). Estudo retrospectivo de neoplasmas do sistema digestório em caninos. Programa de Pós-Graduação em Veterinária.

Myers III, N. C., \& Penninck, D. G. (1994). Ultrasonographic diagnosis of gastrointestinal smooth muscle tumors in the dog. Veterinary Radiology \& Ultrasound, 35(5), 391-397. https://doi.org/10.1111/j.1740-8261.1994.tb02059.x.

Penninck, D. G. (2004). Trato gastrointestinal. In T. . Nyland \& J. S. Matton (Eds.), Ultrassom diagnóstico em pequenos animais (pp. 211-234). Roca, Brasil.

Penninck, D. G., \& D’Anjou, M. A. (2011). Atlas de ultrassonografia de Pequenos animais (p. 513p.). Guanabara Koogan.

Wronski, M., Cebulski, W., Slodkowski, M., \& Krasnodebski, I. W. (2009). Gastrointestinal stromal tumors: ultrasonographic spectrum of the disease. Journal of Ultrasound in Medicine, 28(7), 941948. https://doi.org/10.7863/jum.2009.28.7.941.

\section{Histórico do artigo:}

Recebido: 27 de dezembro de 2020

Aprovado: 22 de fevereiro de 2021

Disponível online: 16 de março de 2021
Licenciamento: Este artigo é publicado na modalidade Acesso Aberto sob a licença

Creative Commons Atribuição 4.0 (CC-BY 4.0), a qual permite uso irrestrito, distribuição, reprodução em qualquer meio, desde que o autor e a fonte sejam devidamente creditados 